\title{
The Effects of Perceived Discrimination on Ambulatory Blood Pressure and Affective Responses to Interpersonal Stress Modeled Over 24 Hours
}

\author{
Laura Smart Richman \\ Duke University
}

\author{
Elizabeth Pascoe \\ Duke University
}

\author{
Jolynn Pek \\ University of North Carolina at Chapel Hill \\ Daniel J. Bauer \\ University of North Carolina at Chapel Hill
}

\begin{abstract}
Objective: This research examined the impact of perceived discrimination on ambulatory blood pressure (ABP) and daily level affect during social interaction. Design: For 24 hrs, adult Black and White participants wore an ABP monitor and completed palm pilot diary entries about their social interactions. Main Outcome Measures: Mean level and time-trend trajectories of blood pressure and heart rate were examined as well as mean level measures of positive and negative affect after stressful and nonstressful social interactions. Results: Analyses showed that, after controlling for important covariates, perceived discrimination predicted the slopes of both wake and nocturnal ABP responses, with those who reported more discrimination having steeper daytime trajectories for systolic and diastolic blood pressure and less nighttime dipping in heart rate over time as compared to those who had reported relatively infrequent discrimination. High levels of perceived discrimination were also related to positive and negative affective responses after stressful encounters. Conclusions: These results suggest that, regardless of race, perceived discrimination is related to cardiovascular and affective responses that may increase vulnerability to pathogenic processes.
\end{abstract}

Keywords: perceived discrimination, ambulatory blood pressure, affect

Experiences with various forms of unfair treatment have important implications for physical and mental health (Harrell, Hall, \& Taliaferro, 2003; Jackson, Kubzansky, \& Wright, 2006; Pascoe \& Smart Richman, 2009; Williams, Neighbors, \& Jackson, 2003). A recent meta-analysis on perceived discrimination and health (Pascoe \& Smart Richman, 2009) finds support for different forms of discrimination (e.g., racial, gender, sexual, and general unfair treatment) being related to elevated blood pressure, heart rate (HR), and cortisol secretions in laboratory studies. These studies incorporate a range of methodologies to measure the effects of discrimination such as speech or writing tasks in which participants recall past experiences, reactions to videotaped or audiotaped discriminatory scenarios or inferred discrimination based on purported views of the experimenter, and actual discrimination delivered by an experimenter or confederate of the study. Collectively, these studies suggest that physiological responses to discrimina-

Laura Smart Richman, Department of Psychology and Neuroscience, Duke University; Jolynn Pek, Department of Psychology, University of North Carolina at Chapel Hill; Elizabeth Pascoe, Department of Psychology and Neuroscience, Duke University; and Daniel J. Bauer, Department of Psychology, University of North Carolina at Chapel Hill.

Acknowledgements: This work was supported by Grant 1 K01-MH074942-02 from the National Institutes of Mental Health awarded to the first author. We gratefully acknowledge the help of Alana Genderson for data collection and James Lane for programming of the palm pilots.

Correspondence concerning this article should be addressed to Laura Smart Richman, Department of Psychology and Neuroscience, Duke University, Box 90085, Flowers Drive, Durham, NC 27708. E-mail: 1richman@duke.edu tion are similar to how people respond to other kinds of uncontrollable and unpredictable stressful events.

The stress literature and recent work specifically on the stress incurred by discrimination (Ong, Fuller-Rowell, \& Burrow, 2009) points to the enduring effects of past discrimination on how day-to-day interactions are experienced. Chronic experiences of stress can affect how people appraise future stressful events and may increase the tendency to experience and respond more acutely to daily stressors. In a daily diary study by Ong et al. (2009), individuals who reported more chronic racial discrimination also reported higher levels of daily negative affect, anxiety, and depression. Other research finds that experience with discrimination is associated with cardiovascular dysregulation, marked by more acute cardiovascular responses (CVRs) to interpersonal stress and slower recovery to baseline, suggesting that repeated exposure to discrimination may work in ways that prepare the body to be more physically reactive in stressful or potentially stressful social situations (Guyll, Matthews, \& Bromberger, 2001; Smart Richman et al., 2007).

The importance of research on the impact of discrimination experiences lies in the evidence that heightened physiological responses to stress can, over time, have downstream effects on health. An elevated CVR to stress has been linked to the development of coronary heart disease and hypertension (Manuck \& Krantz, 1986). Longitudinal research that tracks normotensive individuals for 20 years or more shows that the extent of blood pressure responses to stress-inducing manipulations were predictive of an increased risk for the development of essential hypertension (Treiber et al., 2003). 
To date, the research examining the relationship between perceived discrimination and elevated cardiovascular responding has been focused primarily on establishing that such an association exists. Few studies have examined pathways by which discrimination can incur its harmful effects. Physiological mechanisms, such as experiences with racism and nocturnal blood pressure recovery, suggest that perceived racism increases the risk of nondipping at nighttime (as indicated by a change from waking to nighttime blood pressure of $10 \%$ or more; Brondolo et al., 2008). The typical trajectory of blood pressure throughout the day is that the lowest point is in the early morning and the peak is in the mid-afternoon. The association of racism with a diminished decline in blood pressure at nighttime is important because such nondipping is associated with an increased risk for hypertension and cardiovascular disease (see Bishop, Pek, \& Ngau, 2006).

Another central factor to consider is whether experiences with discrimination can influence the ways in which individuals affectively respond to social interactions. A large body of literature now exists establishing that the tendency to experience positive emotion is related to better health outcomes (e.g., Pressman \& Cohen, 2005; Smart Richman et al., 2005). One way in which positive emotions may function is as a stress buffer, by decreasing the intensity and speeding the recovery from stressful events (Fredrickson, 1998). Resilient people who seem to "bounce back" - both physiologically and emotionally - from stressful experiences tend to experience more positive emotion even during times of stress (Tugade \& Fredrickson, 2004). Conversely, the relationship between the tendency to experience sustained negative affect and increased risk for a range of disease outcomes is well-documented (e.g., Booth-Kewley \& Friedman, 1987, for a review). If experiences with discrimination are predictive of an increased tendency to have lower positive affect and more negative affect after stressful events, then we can further understand the ways in which perceived discrimination may incur harmful effects by influencing how people affectively respond to social interaction. In addition, an association between perceived discrimination and tendencies to experience positive and negative affect in interpersonal interactions may be an important pathway by which discrimination is related to poor mental health outcomes (e.g., Williams et al., 2003).

Much of the work in this area has been confined to laboratorybased studies that examine physiological and emotional arousal associated with laboratory analogues of discrimination (see Harrell, Hall, \& Taliaferro, 2003, for a review). These studies provide important insights into the responses to discrimination during a narrow time period over the course of an experiment. We sought to extend this research beyond the laboratory to examine how experiences with perceived discrimination may predict CVRs throughout a 24-hr period. The measurement of ambulatory blood pressure $(\mathrm{ABP})$ enables individual's responses to their daily experience to be measured and has important clinical implications because there is evidence that $\mathrm{ABP}$ is more closely associated with cardiovascular morbidity and mortality than clinic blood pressure (see Brondolo et al., 2008). Importantly, modeling ABP over the course of a day allows for an examination of how perceived discrimination can impact physiological and emotional responses to social interaction and the trajectory of these physiological responses. The few studies that have examined the relationship of discrimination and ABP have found a positive association between perceived appearance-related discrimination and waking $\mathrm{ABP}$ among adolescents (Matthews et al., 2005), perceived racism and average daytime systolic blood pressure (SBP) in an adult sample (Steffen, McNeilly, Anderson, \& Sherwood, 2003), and perceived racism and nocturnal blood pressure among Blacks and Latinos (Brondolo et al., 2008). However, the generalizability of these studies is limited by restricted sample age and race. Furthermore, these studies have not modeled time trends to examine how perceived discrimination can impact not just mean-level responses, but also the slopes of these effects. We further sought to extend these findings by having participants report on the stressfulness of their social interactions over the course of a day and their corresponding affective responses to these interactions.

\section{Overview of the Study}

Using a combination of $\mathrm{ABP}$ methodology and palm pilot recordings, we examined how experiences with discrimination affect physiological and emotional responses over the course of a day. We tested the hypothesis that perceived discrimination would predict elevated CVR throughout the day, marked by steeper daytime slopes and decreased nighttime dipping, even if individuals were normotensive and in good health. Furthermore, we examined how affective responses to stressful versus nonstressful interactions were predicted by discrimination. We hypothesized that perceived discrimination would be related to lower positive and higher negative affect after stressful social interactions. We also sought to establish, consistent with prior research (Brondolo et al., 2008), that these effects are not a function of global cynical or neurotic views on the world that may inflate the reporting of discrimination and may also be related to CVR and affective responses.

\section{Method}

\section{Participants}

A community sample of 70 participants was recruited via advertisements and flyers distributed in public places (bus stops, shopping malls, grocery stores) and academic buildings. Because of measurement error (e.g., excessive movement or slippage of the blood pressure cuff) or inability to obtain consistent blood pressure for other reasons, seven participants had large amounts of missing or unreliable data and were excluded from the sample. An additional participant was excluded because of missing data on all the covariates. There remained 31 Black and 31 White participants whose ages ranged from 18 to 53 years. All potential participants were screened over the phone to determine if they met eligibility requirements of being between the ages of 18 and 55, selfidentifying as Black or African American or White, and not taking cardiovascular medication or having cardiovascular disease. The majority of participants (42) were not on medication while the remaining 20 were on medication not known to affect cardiovascular function. There were 36 nonsmokers, 4 smokers, and 22 who refused to answer. Additional sample characteristics are provided in Table 1. Participants were paid between $\$ 80$ and $\$ 100$ based on their timely completion of diary measures after cuff inflations. 
Table 1

Sample Characteristics Across All Participants and by Race

\begin{tabular}{|c|c|c|c|c|c|c|c|}
\hline & \multicolumn{2}{|c|}{ Total $(N=63)$} & \multicolumn{2}{|c|}{ White $(n=31)$} & \multicolumn{2}{|c|}{ Black $(n=32)$} & \multirow[b]{2}{*}{$t(d f=61)$} \\
\hline & $M$ & $S D$ & $M$ & $S D$ & $M$ & $S D$ & \\
\hline \multicolumn{8}{|l|}{ Characteristic } \\
\hline Age & 27.19 & 10.18 & 25.87 & 9.00 & 28.47 & 11.21 & -1.01 \\
\hline Male $(\%)$ & 0.35 & & 0.42 & & 0.28 & & 1.14 \\
\hline High SES $(\%)$ & 0.54 & & 0.68 & & 0.41 & & $2.21^{*}$ \\
\hline Body mass index & 25.48 & 4.94 & 23.64 & 3.87 & 27.26 & 5.26 & $-3.10^{* * *}$ \\
\hline Perceived discrimination & 1.98 & 0.59 & 1.73 & 0.38 & 2.24 & 0.65 & $-3.82^{* *}$ \\
\hline Cook Medley Hostility & 11.93 & 4.51 & 10.52 & 3.65 & 13.45 & 4.87 & $-2.69^{* * *}$ \\
\hline Neuroticism & 94.40 & 9.13 & 95.85 & 7.61 & 93.22 & 10.43 & 1.02 \\
\hline \multicolumn{8}{|c|}{ Average time-varying covariates } \\
\hline Activity (\%) & 0.36 & & 0.26 & & 0.33 & & -1.47 \\
\hline Stressful interactions (\%) & 0.06 & & 0.07 & & 0.05 & & 1.08 \\
\hline Positive affect & 62.98 & 10.30 & 62.94 & 8.48 & 63.46 & 11.89 & -0.20 \\
\hline Negative affect & 17.64 & 13.51 & 15.03 & 10.90 & 20.37 & 15.60 & -1.56 \\
\hline \multicolumn{8}{|l|}{ Average waking measures } \\
\hline $\mathrm{SBP}(\mathrm{mmHg})$ & 125.83 & 14.17 & 123.85 & 13.57 & 127.74 & 14.69 & -1.09 \\
\hline DBP $(\mathrm{mmHg})$ & 73.87 & 7.74 & 72.39 & 7.78 & 75.31 & 7.54 & -1.51 \\
\hline \multirow[t]{2}{*}{ HR (bpm) } & 79.11 & 9.18 & 76.77 & 7.89 & 81.38 & 9.88 & $-2.04^{*}$ \\
\hline & \multicolumn{2}{|c|}{ Total $(N=59)$} & \multicolumn{2}{|c|}{ White $(n=29)$} & \multicolumn{2}{|c|}{ Black $(n=30)$} & $t(d f=57)$ \\
\hline Average sleeping measures & & & & & & & \\
\hline $\mathrm{SBP}(\mathrm{mmHg})$ & 111.32 & 15.59 & 107.69 & 14.69 & 114.82 & 15.88 & -1.79 \\
\hline $\mathrm{DBP}(\mathrm{mmHg})$ & 61.76 & 10.09 & 58.69 & 7.89 & 64.73 & 11.17 & $-2.39^{*}$ \\
\hline HR (bpm) & 66.03 & 9.57 & 61.36 & 7.95 & 90.54 & 8.94 & $-4.16^{* * *}$ \\
\hline
\end{tabular}

Note. $\quad \mathrm{SES}=$ socioeconomic differences; $\mathrm{SBP}=$ systolic blood pressure; $\mathrm{DBP}=$ diastolic blood pressure; $\mathrm{HR}=$ heart rate; bpm $=$ beats per minute $t$-tests were conducted with the assumption of equal variances between Blacks and Whites.

${ }^{*} p<.05 . \quad{ }^{* * *} p<.001$.

\section{Measures}

Demographics. Participants provided information about their income and education. These were combined to form a binary variable of socioeconomic status (SES). Body mass index (BMI) was obtained by measuring height and weight of participants before ABP monitoring.

Perceived discrimination. We assessed perceived discrimination with a 10-item version of the everyday discrimination scale that measures the frequency of experiencing a variety of forms of everyday interpersonal mistreatment over the previous 12 months (Williams, Yu, Jackson, \& Anderson, 1997). A sample item is, "People act as if they are better than you." The original scale contains 9 items and our scale included the additional item of, "People ignore you or act as if you are not there" based on Matthews et al. (2005). The items were scored along a 4-point scale $(1=$ never, $2=$ rarely, $3=$ sometimes, $4=$ often $)$ with higher scores reflecting higher levels of discrimination.

Hostility. Dispositional hostility was measured with the Cook-Medley Hostility (Ho) Scale (Cook \& Medley, 1954) with 27 items rated as either true (1) or false (0). Following recommendations made by Barefoot, Dodge, Peterson, Dahlstrom, and Williams (1989), the analysis is based on Ho scores which are sums of the subscale scores of cynicism, hostile affect, and aggressive responding. Higher scores reflect higher levels of trait hostility.

Neuroticism. Forty items answered on a Likert scale ranging from strongly disagree (0) to strongly agree (4) from the neurot- icism subscale of the NEO-PIR (Costa \& MacCrae, 1992) were used. Higher scores reflect a higher enduring tendency to experience negative emotional states.

Diary questions. Participants were asked to indicate their current affective state using a visual analog scale ranging from 1 to 100 , with higher scores reflecting greater intensity of emotion. Based on exploratory factor analyses, scores for positive affect were computed by taking the mean of the responses on happy, interested, pleased, and bored (reversed). Similarly, negative affect was computed by taking the mean of sad, angry, anxious, resentful, and helpless. Other diary questions asked participants about whether they were in a social interaction $(0=n o, 1=y e s)$, and whether the interaction was stressful $(0=n o, 1=y e s)$ and their posture (on feet, sitting, or lying down) at that moment.

\section{Procedure}

Participants were scheduled to complete all parts of the study during a typical work day. Participants first came to the lab to complete the baseline psychosocial questionnaires on a computer. The next day, they were equipped with the AccuTrackerII ABP monitor (SunTech Medical, Raleigh, NC) in the morning before 10:00 a.m. and were instructed to go about their normal daily routines while wearing the monitor for approximately $24 \mathrm{hr}$. Participants answered a series of questions on a PalmOne Zire31 handheld computer within $5 \mathrm{~min}$ of each daytime blood pressure reading. They returned to the lab the next morning to have the monitors removed and the data downloaded. Blood pressure and 
HR measurements were programmed to be taken at variable intervals three times an hour during participants' waking periods. During sleep, readings were taken every hour. AccuTrackerII software automatically flagged potential artifacts in the data and these readings were each evaluated by at least two independent judges and, if warranted, were removed before data analyses.

Statistical analyses overview. Random coefficients regression models were fitted using PROC MIXED (Littell et al., 1996). All models were estimated with restricted maximum likelihood, and a spatial power error structure (autoregressive order which accounts for unequally spaced time intervals) was specified to account for the autocorrelation among the sequentially assessed measures within participants. Continuous person level variables (age, BMI, hostility, and neuroticism) were grand mean centered before they were entered into the models. Binary variables of race $($ Black $=1$, White $=0)$, sex $($ female $=1$, male $=0)$, and SES (high $=1$, low $=0$ ) were coded to indicate referent variables of White men with low socioeconomic standing. The following timeinvariant covariates were entered into each model: sex, race, the interaction of sex by race, SES, age, BMI, hostility, and neuroticism. The categorical time-varying covariate of posture was coded in reference to sitting because this was the dominant posture in the course of participants' time awake. Model diagnostics identified an outlier that was removed in the final models leaving a sample of $N=61$.

A two-rate model with separate slopes for sleep and wake periods was used to express the dynamics of cardiovascular assessment as a function of these two time phases. Time was coded to reflect hours and was centered at time of last waking moment to scale participants' diurnal CVRs to a common wake/sleep cycle. A random intercept for the outcome measure at time of sleep and random slopes for each linear piece were also specified, and an unconstrained variance-covariance matrix of random effects was estimated. As a comparison to more traditional models examining mean level differences of CVRs throughout a day, an additional model was fit to the data with a binary variable (wake) that distinguished between awake and asleep periods with only random intercepts specified. Interactions of sex, race, and perceived discrimination with wake were entered into the models to examine their effects on mean levels of CVRs during the distinct phases of wake and sleep. Nighttime dipping was conceptualized as the rate of change (decrease) in SBP, diastolic blood pressure (DBP), and HR across participants' time asleep. There were no strict categories of dipping and participants' slopes of dipping were allowed to vary randomly.

Finally, we examined how perceived discrimination may be associated with heightened negative affect and decreased positive affect after stressful social interactions throughout the course of a day. In these models, only measures taken during time awake were examined and random intercepts and slopes were specified. The variables entered into the model were sex, race, the interaction of sex and race, hostility, neuroticism, perceived discrimination, continuous time, and time-varying reports of stress. SES was not significant in predicting emotions and was removed from the final models. Stress was decomposed into between-person and withinperson effects by person-mean centering (cf. Schwartz \& Stone, 1998) and were both added into the model. By doing this, we were able to take advantage of the richness of the ecological momentary assessment data and examine not only whether people who have a higher tendency of reporting stressful events (between-person stress) are more likely to experience less positive and more negative affect as their levels of discrimination increased, but also whether perceived discrimination predicted decreased positive affect and increased negative affect after stressful events (withinperson stress). All possible interactions among between-person stress, within-person stress, and perceived discrimination were examined. Negative emotion was log transformed to correct for positive skewness.

\section{Results}

\section{Participant Characteristics}

Sample characteristics are presented in Table 1. Participants provided $60.52(S D=11.17)$ usable ambulatory readings and $31.98(S D=11.03)$ diary entries across the 24-hr monitoring period. Four participants did not have data during the sleep period.

\section{Time-Trend Effects of Sex, Race, and Perceived Discrimination}

Overall, there were significant time trends on SBP, DBP, and HR as presented in Table 2. All three outcomes had significant negative slopes at time of sleep, indicating an expected dipping effect. The time-trend effects of sex and race were nonsignificant for the three outcomes (see Table 2). However, the effect of perceived discrimination and time awake were significant for SBP and DBP such that those with high perceived discrimination tend to exhibit a steeper climbing trend during wake periods compared to those low on perceived discrimination. Simple slope analyses (cf. Preacher, Curran, \& Bauer, 2006) confirmed that participants with low perceived discrimination had significant negative slopes for SBP and DBP while awake, $t(54.5)=-0.27, p<.05$ and $t(51.6)=-.05, p<.0001$, respectively (see Figure 1 with the three panels indicating the three cardiovascular outcomes measured over 7:00 a.m. until 11:00 a.m. [or $35 \mathrm{hr}$ ] the next day). During sleep, the effect of perceived discrimination by time on HR was marginally significant but should be interpreted in the presence of small sample size since there was one reading taken at sleep for every three readings taken awake. Simple slope analyses confirmed that both low and high perceived discrimination were associated with significant negative slopes, $t(48)=-6.35, p<$ .0001 and $t(29.5)=-4.03, p<.01$ although the slope for low perceived discrimination $(-2.57)$ was much larger in magnitude than high perceived discrimination $(-1.48)$. There were significant random effects for all three measures (see Table 1), suggesting much between-person variability at time of sleep and rate of dipping for SBP, DBP, and HR. Comparable mean level analyses examining binary time did not obtain any significant results of interest.

\section{Predictors of Daily Emotion}

We examined whether experiences throughout the day could provide some insight into the physiological effects that were found. Similar to cardiovascular outcomes, the random effects for positive and negative affect were significant, indicating that there 
Table 2

Estimates (SEs) of Time and Perceived Discrimination Effects on $A B P(N=61)$

\begin{tabular}{|c|c|c|c|}
\hline & SBP & DBP & HR \\
\hline \multicolumn{4}{|l|}{ Fixed effects } \\
\hline Intercept & $122.43(5.23)^{* * * *}$ & $68.66(2.96)^{* * *}$ & $73.26(3.35)^{* * * *}$ \\
\hline Female & $-6.86(5.62)$ & $0.85(3.19)$ & $0.08(3.61)$ \\
\hline Black & $4.30(6.62)$ & $1.56(3.75)$ & $-0.53(4.25)$ \\
\hline Female $\times$ Black & $-0.85(6.93)$ & $-2.30(3.85)$ & $6.58(4.33)$ \\
\hline High SES & $8.30(3.36)^{*}$ & $2.71(1.86)$ & $-0.83(2.09)$ \\
\hline Age & $0.17(0.19)$ & $0.43(0.10)^{* * * *}$ & $0.18(0.12)$ \\
\hline BMI & $0.97(0.42)^{*}$ & $0.38(0.23)$ & $0.24(0.26)$ \\
\hline Hostility & $-0.33(0.46)$ & $0.04(0.25)$ & $0.11(0.28)$ \\
\hline Neuroticism & $-0.01(0.20)$ & $0.07(0.11)$ & $-0.22(0.13)$ \\
\hline On feet & $3.43(0.49)^{* * * *}$ & $3.17(0.42)^{* * *}$ & $11.20(0.52)^{* * * *}$ \\
\hline Lying down & $-4.90(0.93)^{* * * *}$ & $-6.56(0.78)^{* * * *}$ & $-3.35(0.96)^{* * *}$ \\
\hline Time awake & $-0.24(0.15)$ & $-0.47(0.14)^{* *}$ & $-0.11(0.20)$ \\
\hline Time asleep & $-3.10(0.68)^{*}$ & $-1.69(0.40)^{* * * *}$ & $-2.03(0.46)^{* * * *}$ \\
\hline Perceived discrimination & $4.92(4.36)$ & $3.54(2.50)^{*}$ & $3.06(2.84)$ \\
\hline Female $\times$ time wake & $0.29(0.17)$ & $0.34(0.16)$ & $-0.12(0.22)$ \\
\hline Female $\times$ time sleep & $0.62(0.82)$ & $-0.32(0.49)$ & $0.46(0.57)$ \\
\hline Black $\times$ time wake & $-0.01(0.18)$ & $-0.19(0.17)$ & $0.19(0.24)$ \\
\hline Black $\times$ time sleep & $-0.30(0.85)$ & $0.23(0.52)$ & $-0.58(0.60)$ \\
\hline Perceived discrimination $\times$ time wake & $0.37(0.16)^{*}$ & $0.33(0.14)^{*}$ & $0.05(0.20)$ \\
\hline Perceived discrimination $\times$ time sleep & $0.01(0.71)$ & $-0.06(0.43)$ & $0.93(0.50)^{\dagger}$ \\
\hline \multicolumn{4}{|l|}{ Random effects } \\
\hline Intercept & $199.02(44.95)^{* * * * *}$ & $61.87(14.68)^{* * * *}$ & $77.58(19.66)^{* * *}$ \\
\hline Slope awake & $0.08(0.07)$ & $0.15(0.06)^{* *}$ & $0.37(0.12)^{* * *}$ \\
\hline Slope asleep & $4.65(1.59)^{* * *}$ & $1.19(0.58)^{*}$ & $1.51(0.75)^{*}$ \\
\hline Intercept, slope awake & $3.62(1.56)^{*}$ & $2.00(0.80)^{* * *}$ & $2.47(1.26)^{*}$ \\
\hline Intercept, slope asleep & $-10.43(6.14)$ & $-3.36(2.18)$ & $-7.94(3.21)^{*}$ \\
\hline Slope awake, slope asleep & $-0.18(0.25)$ & $-0.10(0.15)$ & $-0.23(0.22)$ \\
\hline Residual & $114.48(4.56)^{* * * *}$ & 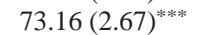 & $112.75(3.98)^{* * * * *}$ \\
\hline Spatial power multiplier & $0.09(0.02)^{* * * *}$ & $0.04(0.01)^{* * * *}$ & $0.05(0.01)^{* * * *}$ \\
\hline
\end{tabular}

Note. $\mathrm{SBP}=$ systolic blood pressure; $\mathrm{DBP}=$ diastolic blood pressure; HR $=$ heart rate; and $\mathrm{SES}=$ socioeconomic status.

${ }^{*} p<.05 .{ }^{* * *} p<.001 .{ }^{* * *} p<.0001 .^{\dagger} p=.069$.

was high variability in the intercepts and slopes of emotions across the day.

Non-stressful situations were associated with reports of higher positive affect than stressful situations $(b=6.71$, S.E. $=1.62, p<$ .0001). For positive affect, there was a significant interaction among between-person and within-person reports of stressful events, $t(1,1599)=-3.01, p<.01$ and a marginally significant three-way interaction between perceived discrimination and these two measures of stress, $t(1,1,599)=-1.90, p=.058$. The three-way interaction is presented in the two panels of Figure 2. For participants who reported many stressful events, high perceived discrimination was associated with low positive affect after stressful situations whereas perceived discrimination did not moderate the effect of a nonstressful situation on reports of positive affect. Among participants low on between-person stress, higher perceived discrimination was associated with lower positive affect regardless of whether situations were deemed stressful or nonstressful. These results suggest that for positive affect, the effect of perceived discrimination is manifest only in stressful situations when between-person stress levels are high.

Similarly, main effects of between-person stress events and time-varying reports of stress were predictive of more negative emotions, $t(1,51.6)=2.88, p<.01$ and $t(1,1654)=8.33, p<$ .0001 , respectively. Although the three-way interaction between perceived discrimination, between-person stress and within-person stress was not significant-likely owing to the low reports of stressful events (see Table 1) - the two-way interactions of perceived discrimination by between-person stress and perceived discrimination by within-person stress were significant, $t(1,52.6)=$ $2.63, p<.01$ and $t(1,1623)=-3.26, p<.01$, respectively. These two interactions are presented in Figures 3 and 4, respectively. In Figure 3, for participants who tended to report experiencing a greater number of stressful events, high perceived discrimination was associated with high negative affect. A trait-level interaction was observed; in general, participants who had a higher tendency to classify social interactions as stressful experienced more negative emotions as their levels of perceived discrimination increased. However, contrary to predictions, in the context of social interactions, as depicted in Figure 4, increasing levels of perceived discrimination were associated with decreased negative affect during a stressful episode. In nonstressful situations, negative affect was not moderated by perceived discrimination.

\section{Discussion}

The results of this research suggest that perceived discrimination has important implications for cardiovascular and affective responding over the course of a $24-\mathrm{hr}$ period. Consistent with our hypotheses, we found that reports of past discrimination were 

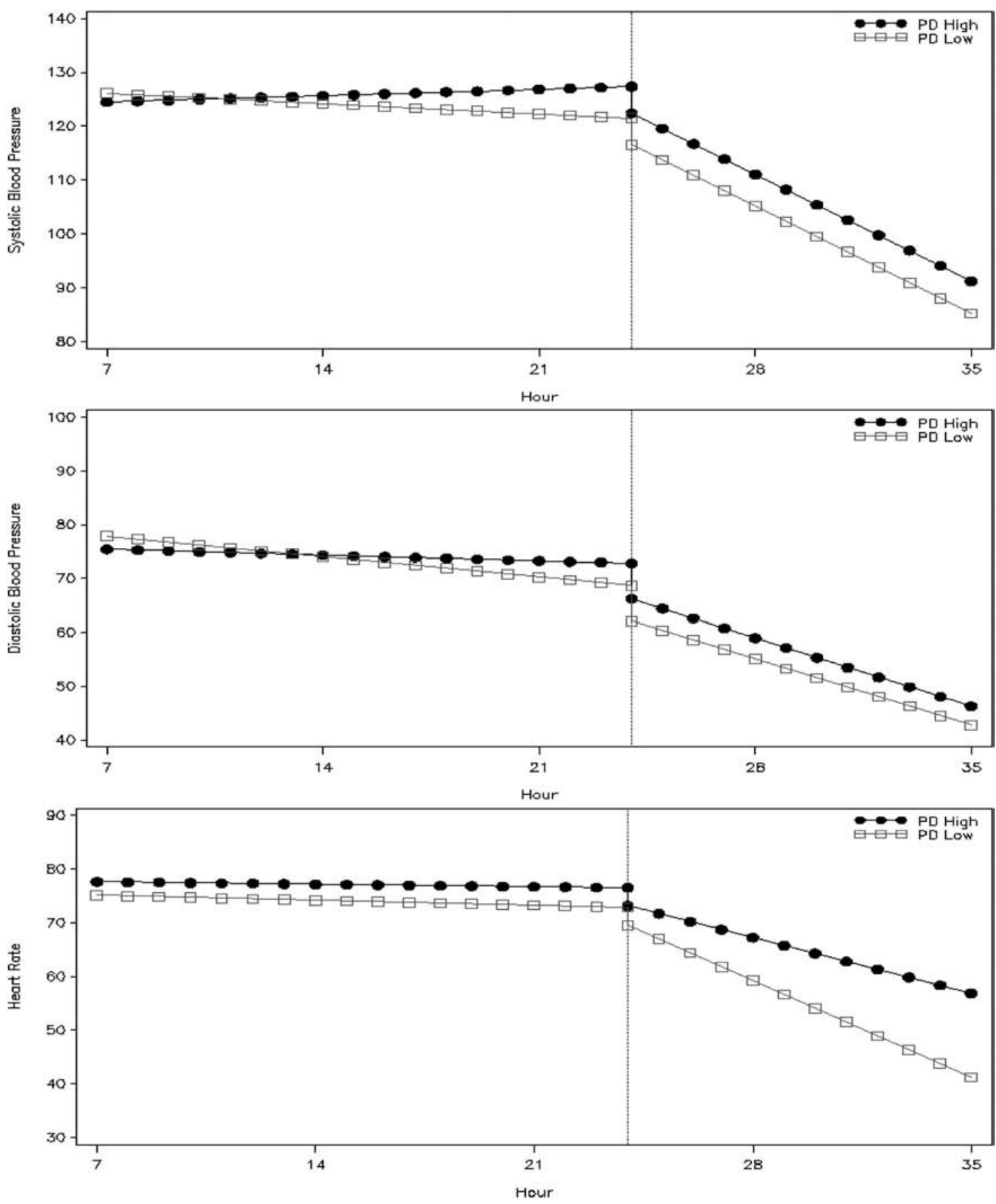

Figure 1. Effect of time and perceived discrimination on ambulatory cardiovascular measures. High and low values of perceived discrimination (PD) reflect $\pm 1 S D$ from the mean value observed in the sample (cf. Aiken \& West, 1991) and the vertical dashed line refers to participants' time of sleep and the discontinuities in the slopes for each level of PD reflect postural differences (sitting vs. lying) in the outcomes.

associated with steeper trajectories of SBP and DBP throughout the day and decreased nocturnal dipping in HR. Our diary data on how people reacted to their interpersonal interactions also revealed potential ways in which we can understand the enduring effects of perceived discrimination on health. We found that perceived discrimination was related to affective responses after interpersonal interactions. For those people who reported many stressful events throughout the day, high perceived discrimination was associated with low positive affect after stressful situations. Similarly, people who had a high frequency of stressful events were more likely to experience negative affect as their reported discrimination levels increased. 

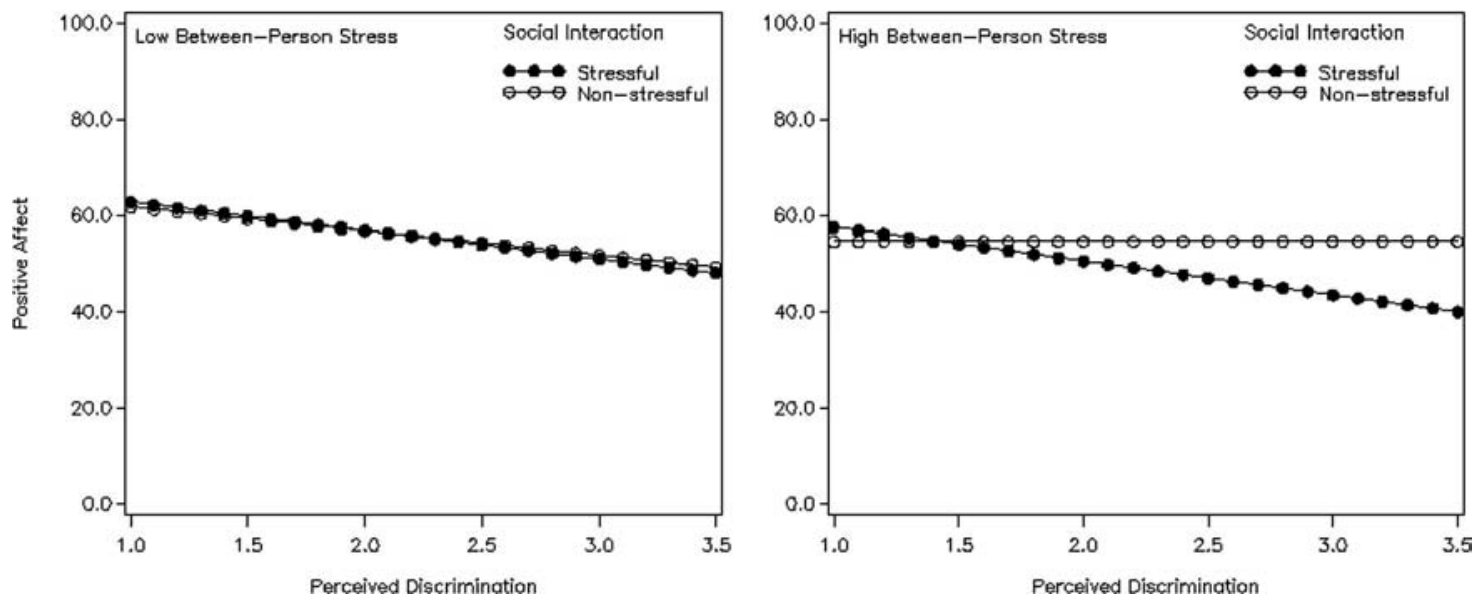

Figure 2. Effect of perceived discrimination, between-person levels of stress reporting and time-varying indications of stressful situations on positive affect. Low and high levels of between-person stress reflect $\pm 1 S D$ from the mean value observed in the sample (cf. Aiken \& West, 1991) as presented in the left and right panels. Stressful and nonstressful time varying reports of social interactions reflect the average minimum $(-0.06)$ and maximum (0.57) across persons after person-mean centering.

The association between perceived discrimination and heightened physiological arousal throughout the day and decreased nighttime dipping suggests that, consistent with previous literature, discrimination may exert its influence by preparing the body to be more physically reactive to stressful experiences (Smart Richman et al., 2007). Over time, such heightened reactivity may increase risk for coronary heart disease and hypertension. The present findings also suggest that the affective responses may be consistent with a model of discrimination stress proliferation - the tendency for stressors to multiply and produce other stressors - that has been

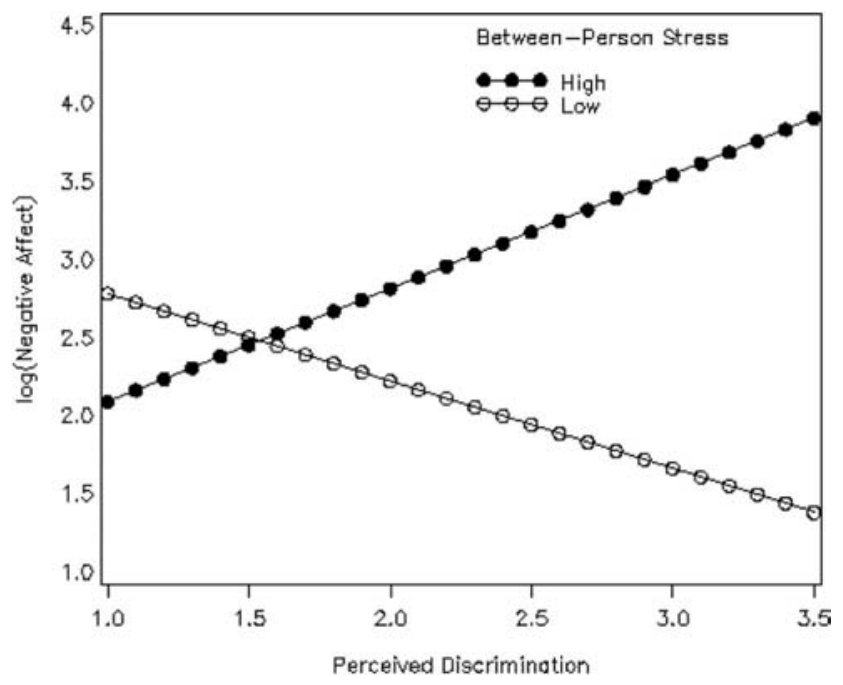

Figure 3. Effect of perceived discrimination and between-person levels of stress reporting on log negative affect. Simple slope analyses confirm that both slopes in the plot are significant; for persons high and low in between-person stress, $t(1,52)=1.98, p<.05$ and $t(1,53)=-2.03, p<$ .05 , respectively. Low and high levels of between-person stress reflect \pm 1 $S D$ from the mean value observed in the sample (cf. Aiken \& West, 1991). recently advanced by Ong et al. (2009). This is a promising area of further inquiry into mediational pathways that link chronic discrimination to psychological distress. Repeated experiences with discrimination may increase vulnerability to respond to stressful social interactions more acutely and to experience more daily negative events, thereby increasing the risk for anxiety and depression.

One strength of this study was that by using multilevel models to analyze ABP recordings and diary data, we were able to have high levels of ecological validity and avoid potential distortions associated with retrospective assessments. The uniqueness of the present analyses also lies in actively modeling time, allowing for

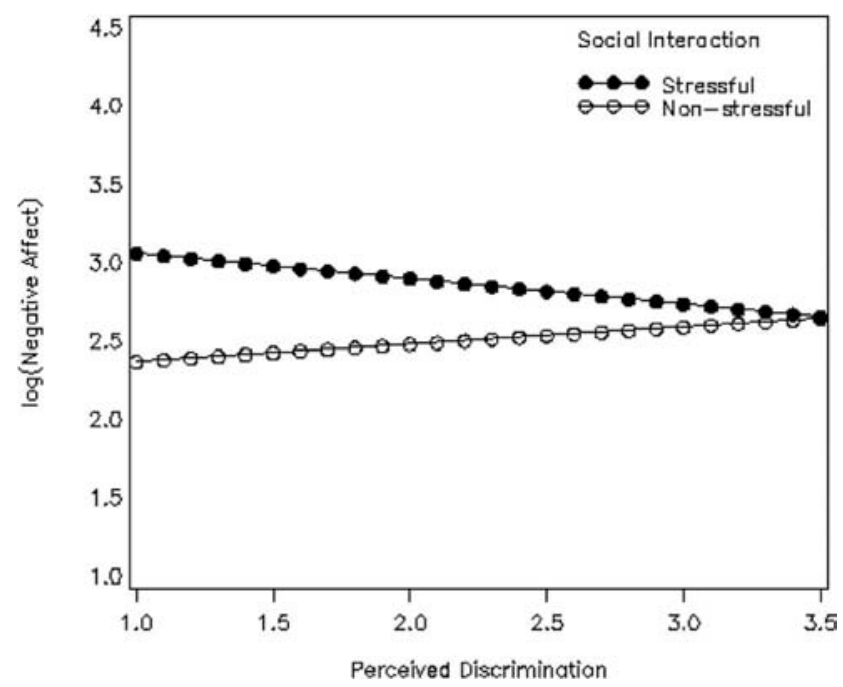

Figure 4. Effect of perceived discrimination and time-varying reports of stressful situations on log negative affect. Stressful and nonstressful time varying reports of social interactions reflect the average minimum $(-0.06)$ and maximum (0.57) across persons after person-mean centering. 
an examination of how perceived discrimination can impact patterns of $\mathrm{ABP}$ over the course of $24 \mathrm{hr}$. In doing so, we were able to replicate the nondipping effects found in previous research (Brondolo et al., 2008) as well as determine that perceived discrimination was positively related to steeper daytime blood pressure slopes.

Although the Black participants in our sample reported more experiences with discrimination, the pattern of physiological and affective responses was similar for both Blacks and Whites. These results are consistent with a comprehensive meta-analysis on discrimination and health that found that the toxic effects of discrimination on both physical and mental health outcomes were not distinguishable based on the type of discrimination experienced (Pascoe \& Smart Richman, 2009). This suggests that similar mechanisms underlie how discrimination is experienced, regardless of what the basis is perceived to be. However, other research has found that certain kinds of discrimination may be more harmful than others. Matthews et al., (2005) found that appearance, but not race-based discrimination was related to elevated ABP among adolescents (Matthews et al., 2005). Based on our findings, we cannot exclude the possibility that certain kinds of discrimination may have more detrimental effects than others.

Importantly also, we controlled for the personality variables of neuroticism and hostility in our models, eliminating a possible interpretation that the reporting of discrimination and the outcomes could be explained by these traits. For example, neuroticism has been related to higher $\mathrm{ABP}$ and more negative and less positive mood on average as compared to those who were low on this trait (Raikkonen, Matthews, Flory, Owens, \& Gump, 1999). A full understanding of how the perceived discrimination impacts physiological and emotional outcomes without controlling for personality variables that can be related to the perception and tendency to report discrimination experiences, can lead to a limitation in interpretability of the effects. It is noteworthy that our effects remained even after taking these personality traits into account.

A limitation of our study was that our sample size did not enable us to have the power to test for possible mediation effects of mood. This leaves open the question of whether positive and negative affective responses to social interaction mediates the CVR trends we found or whether these are separate processes. There is some evidence that positive emotions in general may speed homeostatic processes, specifically for cardiovascular functioning (Fredrickson \& Levenson, 1998) and as a result, positive emotions may reduce stress on the cardiovascular system in the face of negative life events (see also Fredrickson, Mancuso, Branigan, \& Tugade, 2000). Future studies would benefit from examining whether tendencies to experience increased negative mood and decreased positive mood over the course of a day, particularly after stressful social interactions, mediates the effect of perceived discrimination on CVR. In addition, our study is cross-sectional. Future ABP studies that are conducted over a longer period of time, such as over the course of a week or more, would potentially enable an examination of $\mathrm{ABP}$ and affective responses to actual experience of perceived discrimination as well as coping responses to these events.

\section{Conclusions}

Our results suggest that perceived discrimination has harmful effects for Blacks and Whites and that these experiences are associated with a pattern of $\mathrm{ABP}$ responses throughout the course of a day that may pave the way for pathogenic processes associated with heightened physiologic reactivity. Furthermore, perceived discrimination affected the ways in which people responded to stressful interpersonal experiences, by increasing the likelihood of responding to such events with less positive affect and, when people had high stress levels, perceived discrimination was also related to more negative affect throughout the day, suggesting pathways by which discrimination may affect health outcomes.

\section{References}

Aiken, L. S., \& West, S. G. (1991). Multiple regression: Testing and interpreting interactions. Newbury Park, CA: Sage.

Barefoot, J. C., Dodge, K. A., Peterson, B. L., Dahlstrom, W. G., \& Williams, R. B. (1989). The Cook-Medley Hostility scale: Item content and ability to predict survival. Psychosomatic Medicine, 51, 46-57.

Bishop, G. D., Pek, J., \& Ngau, F. (2006). Blunted Cardiovascular Responses to Daytime Activities as Related to Reduced Nocturnal Blood Pressure Decline. Annals of Behavioral Medicine, 31, 248-253.

Booth-Kewley, S., \& Friedman, H. S. (1987). Psychological predictors of heart disease: A quantitative review. Psychological Bulletin, 101, 343362.

Brondolo, E., Libby, D. J., Dentos, E., Thompson, S., Beatty, D. L., Schwartz, J., ... Gerin, W. (2008). Racism and ambulatory blood pressure in a community sample. Psychosomatic Medicine, 70, 49-56.

Cook, W. W., \& Medley, D. M. (1954). Proposed hostility and pharisaicvirtue scales for the MMPI. Journal of Applied Psychology, 38, 414418.

Costa, P. T., \& McCrae, R. R. (1992). NEO-PI-R professional manual. Odessa, FL: Psychological Assessment Resources.

Fredrickson, B. L. (1998). What good are positive emotions? Review of General Psychology: Special Issue: New Directions in Research on Emotion, 2, 300-319.

Fredrickson, B. L., \& Levenson, R. W. (1998). Positive emotions speed recovery from the cardiovascular sequelae of negative emotions. Cog nition and Emotion, 12, 191-220.

Fredrickson, B. L., Mancuso, R. A., Branigan, C., \& Tugade, M. M. (2000). The undoing effect of positive emotions. Motivation and Emotion, 24, 237-258.

Guyll, M., Matthews, K. A., \& Bromberger, J. T. (2001). Discrimination and unfair treatment: Relationship to cardiovascular reactivity among African American and European American women. Health Psychology, $20,315-325$.

Harrell, J. P., Hall, S., \& Taliaferro, J. (2003). Physiological responses to racism and discrimination: An assessment of the evidence. American Journal of Public Health, 93, 243-248.

Jackson, B., Kubzansky, L., \& Wright, R. J. (2006). Linking perceived unfairness to health: The Perceived Unfairness Model. Review of General Psychology, 10, 21-40.

Littell, R. C., Milliken, G. A., Stroup, W. W., \& Wolfinger, R. D. (1996) SAS system for mixed models. Cary, NC: SAS Institute.

Manuck, S. B., \& Krantz, D. S. (1986). Psychophysiologic reactivity in coronary heart disease and essential hypertension. In K. A. Matthews, S. M. Weiss, T. Detre, T. M. Dembroski, B. Falkner, S. B. Manuck, \& R. B. Williams (Eds.), Handbook of stress, reactivity and cardiovascular disease (pp. 11-34). New York: Wiley

Matthews, K. A., Salomon, K., Kenyon, K., \& Zhou, F. (2005). Unfair treatment, discrimination, and ambulatory blood pressure in Black and White adolescents. Health Psychology, 24, 258-265.

Ong, A. D., Fuller-Rowell, T., \& Burrow, A. L. (2009). Racial discrimination and the stress process. Journal of Personality and Social Psychology, 96, 1259-1271. 
Pascoe, E., \& Smart Richman, L. (2009). Perceived discrimination and health: A meta-analytic review. Psychological Bulletin, 135, 531-554.

Preacher, K. J., Curran, P. J., \& Bauer, D. J. (2006). Computational tools for probing interaction effects in multiple linear regression, multilevel modeling, and latent curve analysis. Journal of Educational and Behavioral Statistics, 31, 437-448.

Pressman, S., \& Cohen, S. (2005). Does positive affect influence health? Psychological Bulletin, 131, 925-971.

Raikkonen, K., Matthews, K., Flory, J. D., Owens, J. F., \& Gump, B. (1999). Effects of optimism, pessimism, and trait anxiety on ambulatory blood pressure and mood during everyday life. Journal of Personality and Social Psychology, 77, 104-113.

Schwartz, J. E., \& Stone, A. A. (1998). Strategies for analyzing ecological momentary assessment data. Health Psychology, 17, 6-16.

Smart Richman, L., Bennett, G. G., Pek, J., Siegler, I., \& Williams, R. B. (2007). Discrimination, dispositions, and cardiovascular responses to stress. Health Psychology, 26, 675-83.

Smart Richman, L., Kubzansky, L. D., Maselko, J., Kawachi, I., Choo, P.,
\& Bauer, M. (2005). Positive emotion and health: Going beyond the negative. Health Psychology, 24, 422-429.

Steffen, P. R., McNeilly, M., Anderson, N., \& Sherwood, A. (2003). Effects of perceived racism and anger inhibition on ambulatory pressure in African Americans. Psychosomatic Medicine, 65, 746-750.

Treiber, F. A., Kamarck, T., Schneiderman, N., Sheffield, D., Kapuku, G., \& Taylor, T. (2003). Cardiovascular reactivity and development of preclinical and clinical disease states. Psychosomatic Medicine, 65, 45-62.

Tugade, M. M., \& Fredrickson, B. L. (2004). Resilient individuals use positive emotions to bounce back from negative emotional experiences. Journal of Personality and Social Psychology, 86, 320-333.

Williams, D. R., Neighbors, H. W., \& Jackson, J. S. (2003). Racial/ethnic discrimination and health: Findings from community studies. American Journal of Public Health, 93, 200-208.

Williams, D. R., Yu, Y., Jackson, J. S., \& Anderson, N. B. (1997). Racial differences in physical and mental health: Socioeconomic status, stress and discrimination. Journal of Health Psychology, 2, 335-351.

\section{E-Mail Notification of Your Latest Issue Online!}

Would you like to know when the next issue of your favorite APA journal will be available online? This service is now available to you. Sign up at http://notify.apa.org/ and you will be notified by e-mail when issues of interest to you become available! 